\title{
Quantitative Evaluation of the Catalytic Activity of Dendrimers with Only One Active Center at the Core. Application to the Nitroaldol (Henry) Reaction
}

Aizpea Zubia, Fernando P. Cossío*, Iñaki Morao, Marina Rieumont and Xabier Lopez*

Contribution from the Kimika Fakultatea, Euskal Herriko Unibertsitatea, P.K. 1072,

20080 San Sebastian-Donostia, Spain

Supporting Information

\section{Synthesis}

a. Chemicals: Commercially available compounds were used as purchased without further purification, except for benzaldehyde (see below). All solvents were dried and distilled according to standard protocols ${ }^{1}$.

b. Chromatography: Merck pre-coated $0.25 \mathrm{~mm}$ silica plates containing a $254 \mathrm{~nm}$ fluorescence indicator were used for analytical thin-layer chromatography. Flash chromatography purifications were performed using silica gel 70-230 mesh and AcOEt/Hx mixtures.

c. Spectroscopy: ${ }^{1} \mathrm{H}$ NMR and ${ }^{13} \mathrm{C}$ NMR spectra were recorded on a Varian Gemini 200 (200 MHz) spectrometer. Chemical shifts are reported as $\delta$ values (ppm) with respect to TMS and deuterated chloroform. IR spectra were recorded as thin films on KBr disks on a Perkin Elmer 1600 Series FTIR spectrophotometer connected to a PC. All mass spectra were acquired on a Bruker Biflex TOF mass spectrometer equipped with a nitrogen laser with an emission wavelength of $337 \mathrm{~nm}$. Spectra were obtained in the linear mode at an accelerating voltage of $19 \mathrm{kV}$. Each mass determination was averaged from three sample-standard pairs. For these studies a mixture of 3,5-dihydroxybenzoic acid and tetrahydrofurane was 
used as a matrix. Mass values given throughout the text correspond to $\mathrm{m} / \mathrm{z}$ of the centroid of the envelope of the mass peaks for protonated molecular ions $[\mathrm{M}+\mathrm{H}]^{+}$.

d. Compound preparation and characterization:

\section{[4-(4'-benzyloxy)benzyloxy)]benzyl alcohol:}

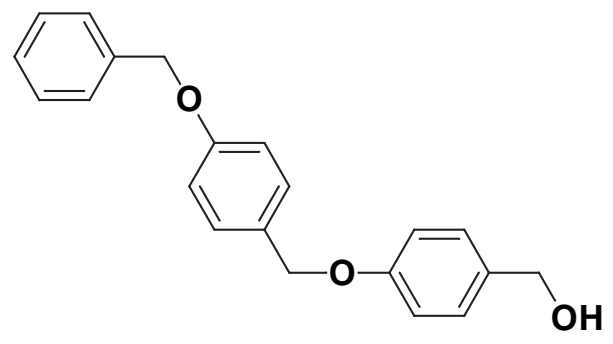

A mixture of 4-hydroxybenzyl alcohol (3.6 g, $29.0 \mathrm{mmol}), 18-\mathrm{C}-6$ crown-ether (0.23 g, $0.86 \mathrm{mmol})$, potassium carbonate $(9.47 \mathrm{~g}, 68.5 \mathrm{mmol})$ and 4-benzyloxybenzyl bromide ${ }^{2}(8.31 \mathrm{~g}, 30.0 \mathrm{mmol})$ in dry acetone $(145 \mathrm{ml})$ was refluxed under argon for $16 \mathrm{~h}$. The mixture was then cooled and evaporated to dryness. The residue was dissolved in water $(150 \mathrm{ml})$ and extracted with $\mathrm{CH}_{2} \mathrm{Cl}_{2}(3 \times 150 \mathrm{ml})$. The extracts were dried with $\mathrm{Na}_{2} \mathrm{SO}_{4}$ and evaporated to dryness. The crude product was purified by column cromatography (AcOEt/Hx 1:5) to give $8.17 \mathrm{~g}(25.5 \mathrm{mmol})$ of a white solid. Yield $=88 \%$. mp: $133-134^{\circ} \mathrm{C}$. ${ }^{1} \mathrm{H}$ NMR ( $\delta$, ppm, $\mathrm{CDCl}_{3}$ ): 7.42-7.24 (m, 9H, arom.), 7.01-6.92 (m, 4H, arom.), $5.08\left(\mathrm{~s}, 2 \mathrm{H}, \operatorname{arCH} \mathrm{H}_{2} \mathrm{O}\right), 4.97$ $\left(\mathrm{s}, 2 \mathrm{H}, \operatorname{arCH}_{2} \mathrm{O}\right), 4.59\left(\mathrm{~d}, \mathrm{~J}=6.0 \mathrm{~Hz}, 2 \mathrm{H}, \operatorname{arCH}_{2} \mathrm{OH}\right), 1.54(\mathrm{t}, \mathrm{J}=6.0 \mathrm{~Hz}, 1 \mathrm{H}, \mathrm{OH}) .{ }^{13} \mathrm{C} \mathrm{NMR}(\delta, \mathrm{ppm}$, $\left.\mathrm{CDCl}_{3}\right): 158.6,158.4,136.8,133.2,129.2,128.6,128.5,127.9,127.4,114.9,69.9,69.7,65.0 . \mathrm{IR}\left(\mathrm{n}, \mathrm{cm}^{-1}\right.$, $\mathrm{KBr}$ ): 3334, 1612, 1518, 1249, 1009. Anal. Calcd for $\mathrm{C}_{21} \mathrm{H}_{20} \mathrm{O}_{3}$ : C, 78.71; H, 6.30. Found: C, 77.88; H, 6.41 .

\section{[4-(4'-benzyloxy)benzyloxy)]benzyl bromide (3):}

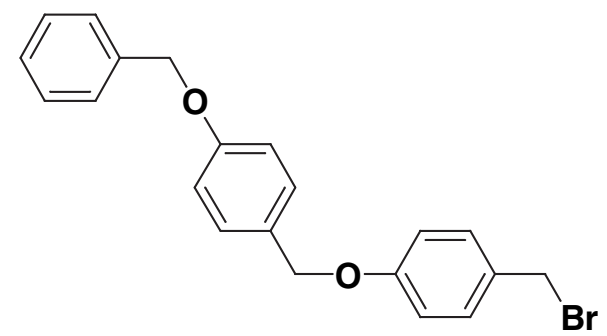

A solution of [4-(4'-benzyloxy)benzyloxy)]benzyl alcohol $(8.17 \mathrm{~g}, 25.5 \mathrm{mmol})$ dissolved in the minimum amount of dry THF (126 ml) was cooled with an ice/water bath. Carbon tetrabromide (12.68 g, $38.25 \mathrm{mmol})$ and triphenylphosphine $(10.0 \mathrm{~g}, 38.25 \mathrm{mmol})$ were added, and the mixture was stirred at room temperature under argon for $1.5 \mathrm{~h}$. Then water $(65 \mathrm{ml})$ was added and the aqueous layer was extracted with $\mathrm{CH}_{2} \mathrm{Cl}_{2}(3 \times 65 \mathrm{ml})$. The combined organic layers were dried $\left(\mathrm{Na}_{2} \mathrm{SO}_{4}\right)$ and evaporated to 
dryness. The crude product was purified by column chromatography with 1:10 ethyl acetate/hexane as eluent to give $7.72 \mathrm{~g}$ of a white solid $(20.1 \mathrm{mmol})$. Yield $=79 \%$. mp: $132-133^{\circ} \mathrm{C} .{ }^{1} \mathrm{H}$ NMR $(\delta, \mathrm{ppm}$, $\mathrm{CDCl}_{3}$ ): 7.44-7.25 (m, 9H, arom.), 7.00-6.89 (m, 4H, arom.), 5.09 (s, $\left.2 \mathrm{H}, \operatorname{arCH}_{2} \mathrm{O}\right), 4.98$ (s, $2 \mathrm{H}, \operatorname{arCh}_{2} \mathrm{O}$ ), 4.47 (s, $\left.2 \mathrm{H}, \operatorname{arCH} \mathrm{CHr}_{2}\right){ }^{13} \mathrm{C} \mathrm{NMR}\left(\delta, \mathrm{ppm}, \mathrm{CDCl}_{3}\right): 158.8,158.6,136.8,130.4,130.0,129.2,128.9,128.5$, 127.9, 127.4, 115.0, 114.9, 69.9, 69.7, 33.9. IR (n, $\left.\mathrm{cm}^{-1}, \mathrm{KBr}\right): 1612,1518,1249,1014$, 747. Anal. Calcd for $\mathrm{C}_{21} \mathrm{H}_{19} \mathrm{O}_{2} \mathrm{Br}$ : C, 65.80; H, 5.01. Found: C, 66.06; H, 4.98.

Synthesis of (3,5-dibenzyloxy)benzyl bromide (4) and [3,5-bis-(3',5'-dibenzyloxy)benzyloxy]benzyl bromide (5): These compounds were prepared following literature procedures ${ }^{3}$.

Synthesis of $(3,4,5$-tribenzyloxy)benzyl bromide (6): This compound was prepared following literature procedures ${ }^{4,5}$.

Methyl [3,4,5-tris(3',4',5'-tribenzyloxy)benzyloxy]benzoate:

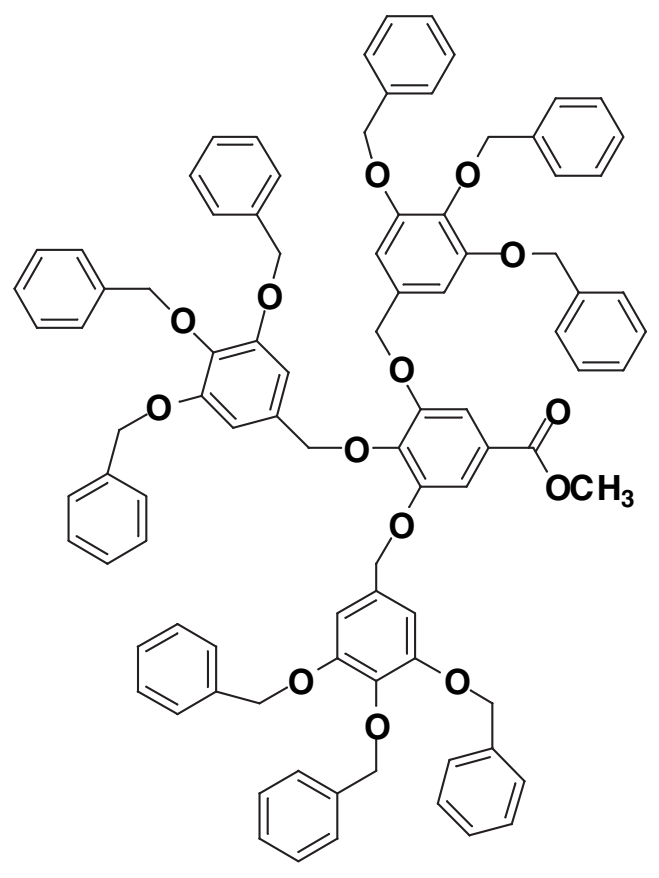

A mixture of methyl 3,4,5-trihydroxybenzoate $(0.53 \mathrm{~g}, 2.9 \mathrm{mmol}), 18-\mathrm{C}-6$ crown-ether $(0.14 \mathrm{~g}, 0.51$ mmol), potassium carbonate $(10.5 \mathrm{~g}, 76.0 \mathrm{mmol})$ and (3,4,5-tribenzyloxy)benzyl chloride ${ }^{4}$ (4.0 g, 9.0 mmol) in anhydrous DMF (55 ml) was refluxed under argon for $24 \mathrm{~h}$. The mixture was then cooled and evaporated to dryness. The residue was dissolved in water $(55 \mathrm{ml})$ and extracted with $\mathrm{CH}_{2} \mathrm{Cl}_{2}(3 \times 55 \mathrm{ml})$. The combined organic layers were dried with $\mathrm{Na}_{2} \mathrm{SO}_{4}$ and evaporated to dryness. The crude product was purified by column chromatography $(\mathrm{AcOEt} / \mathrm{Hx} 1: 1)$ to give $3.31 \mathrm{~g}(2.35 \mathrm{mmol})$ of a white solid. Yield = 81\%. mp: 89-90 ${ }^{\circ} \mathrm{C} .{ }^{1} \mathrm{H}$ NMR ( $\delta$, ppm, $\mathrm{CDCl}_{3}$ ): 7.40-7.25 (m, 45H, arom.), 6.74-6.66 (m, 8H, arom.), 5.02$4.95\left(\mathrm{~m}, 18 \mathrm{H}, \operatorname{arCH}_{2} \mathrm{O}\right), 4.87\left(\mathrm{~s}, 2 \mathrm{H}, \operatorname{arCH}_{2} \mathrm{O}\right), 4.81\left(\mathrm{~s}, 4 \mathrm{H}, \operatorname{arCH}_{2} \mathrm{O}\right), 3.88\left(\mathrm{~s}, 3 \mathrm{H}, \mathrm{CO}_{2} \mathrm{CH}_{3}\right) .{ }^{13} \mathrm{C}^{\mathrm{NMR}}(\delta$, ppm, $\mathrm{CDCl}_{3}$ ): 166.4, 152.9, 152.7, 152.3, 142.2, 138.0, 137.7, 136.8, 132.9, 132.1, 128.3, 128.0, 127.7, 127.6, 127.3, 125.3, 109.4, 107.4, 106,7, 75.1, 74.9, 71.3, 71.0, 70.8, 52.2, 21.0, 14.1. IR (n, $\left.\mathrm{cm}^{-1}, \mathrm{KBr}\right)$ : 
1720, 1593, 1508, 1329, 1230, 1118. Anal. Calcd for $\mathrm{C}_{92} \mathrm{H}_{80} \mathrm{O}_{14}: \mathrm{C}, 78.38 ; \mathrm{H}, 5.73$. Found: C, 77.45; $\mathrm{H}$, 5.76.

\section{[3,4,5-tris(3',4',5'-tribenzyloxy)benzyloxy]benzyl alcohol:}

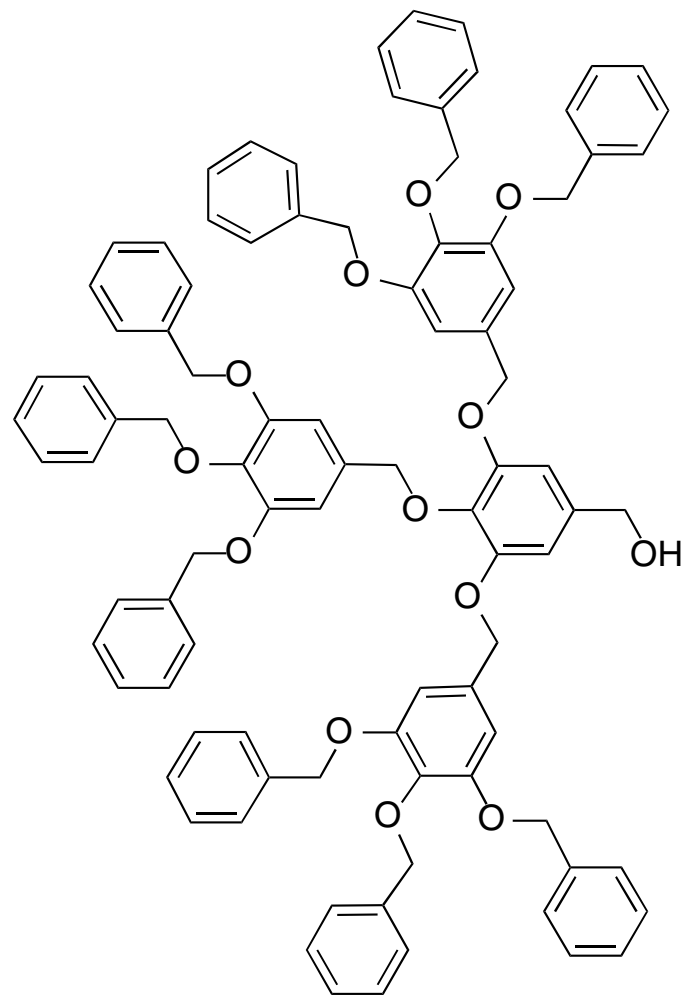

A solution of lithium aluminum hydride $(0.11 \mathrm{~g}, 2.82 \mathrm{mmol})$ in anhydrous ether $(9 \mathrm{ml})$, under an inert atmosphere, was cooled to $0^{\circ} \mathrm{C}$. Methyl [3,4,5-tris(3',4',5'-tribenzyloxy)benzyloxy]benzoate (3.31 g, 2.35 $\mathrm{mmol})$ in anhydrous ether $(9 \mathrm{ml})$ was added dropwise, and the mixture was stirred at room temperature for $3 \mathrm{~h}$. Then, the reaction mixture was cooled with an ice/water bath and treated with water $(0.5 \mathrm{ml})$. The crude was filtered through a Celite pad, and the filtrate was washed with water $(3 \times 9 \mathrm{ml})$. The remaining organic layer was dried over $\mathrm{Na}_{2} \mathrm{SO}_{4}$ and evaporated under reduced pressure, providing $2.82 \mathrm{~g}$ (2.04 mmol) of a white solid. Yield $=87 \%$. m.p.: $130-132^{\circ} \mathrm{C} .{ }^{1} \mathrm{H} \mathrm{NMR}\left(\delta, \mathrm{ppm}, \mathrm{CDCl}_{3}\right): 7.39-7.16(\mathrm{~m}, 45 \mathrm{H}$, arom.), $6.78(\mathrm{~s}$, $2 \mathrm{H}$, arom.), 6.73 (s, 4H, arom.), 6.62 (s, 2H, arom.), 5.00-4.92 (m, 18H, $\left.\operatorname{arCH}_{2} \mathrm{O}\right), 4.87$ (s, 2H, $\left.\operatorname{arCH}_{2} \mathrm{O}\right)$, $4.85\left(\mathrm{~s}, 4 \mathrm{H}, \operatorname{arCH} \mathrm{O}_{2} \mathrm{O}\right), 4.54\left(\mathrm{~d}, 2 \mathrm{H}, \mathrm{J}=6.0 \mathrm{~Hz}, \operatorname{arCH}_{2} \mathrm{OH}\right), 1.19(\mathrm{t}, 1 \mathrm{H}, \mathrm{J}=6.0 \mathrm{~Hz}, \mathrm{OH}) .{ }^{13} \mathrm{C} \mathrm{NMR}(\delta, \mathrm{ppm}$, $\mathrm{CDCl}_{3}$ ): 152.9, 152.8, 152.7, 137.9, 137.8, 137.7, 136.9, 136.8, 133.4, 132.7, 128.4, 128.3, 128.0, 127.9, 127.8, 127.6, 127.4, 127.3, 107.4, 106.7, 106.6, 75.1, 71.2, 71.0, 70.8, 65.1. IR (n, cm $\left.{ }^{-1}, \mathrm{KBr}\right): 3437,1593$, 1503, 1240, 1122. Anal. Calcd for $\mathrm{C}_{91} \mathrm{H}_{80} \mathrm{O}_{13}$ : C, 79.10; H, 5.85. Found: C, 78.42; H, 5.91. 


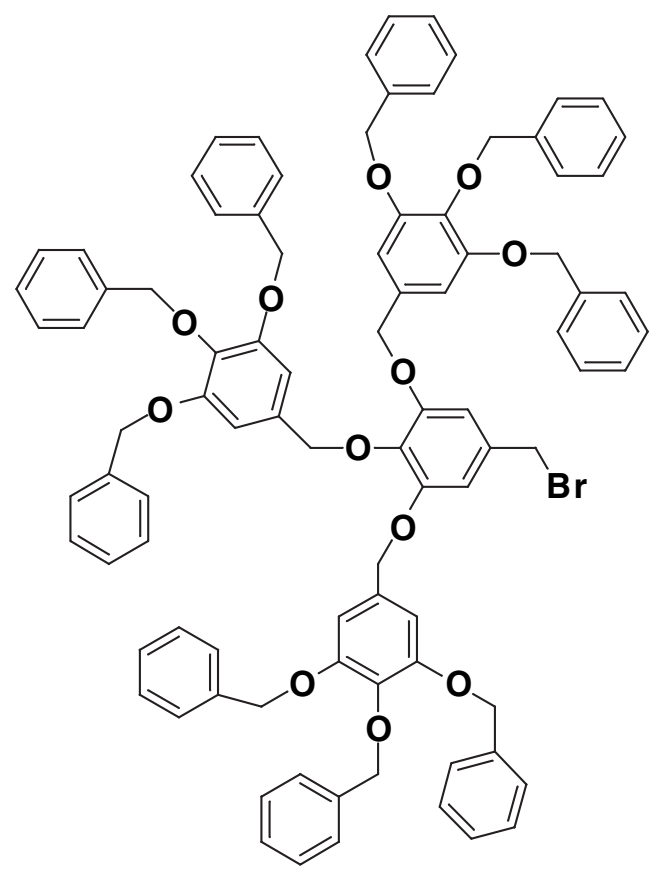

A solution of [3,4,5-tris(3',4',5'-tribenzyloxy)benzyloxy]benzyl alcohol (2.82 g, $2.04 \mathrm{mmol})$ dissolved in the minimum amount of dry THF $(10 \mathrm{ml})$ was cooled with an ice/water bath. Carbon tetrabromide (1.03 $\mathrm{g}, 3.0 \mathrm{mmol})$ and triphenylphosphine $(0.80 \mathrm{~g}, 3.0 \mathrm{mmol})$ were added, and the mixture was stirred at room temperature under argon for $1.5 \mathrm{~h}$. Then water $(5 \mathrm{ml})$ was added and the aqueous layer was extracted with $\mathrm{CH}_{2} \mathrm{Cl}_{2}(3 \times 5 \mathrm{ml})$. The combined organic layers were dried $\left(\mathrm{Na}_{2} \mathrm{SO}_{4}\right)$ and evaporated to dryness. The crude product was purified by column chromatography with 1:10 ethyl acetate/hexane as eluent to give $1.56 \mathrm{~g}$ of a white solid $(1.08 \mathrm{mmol})$. Yield $=53 \%$. m.p.: $125-126^{\circ} \mathrm{C} .{ }^{1} \mathrm{H}$ NMR $\left(\delta, \mathrm{ppm}, \mathrm{CDCl}_{3}\right): 7.40-7.14$ (m, 45H, arom.), 6.74 (s, 2H, arom.), 6.71 (s, 4H, arom.), 6.63 (s, 2H, arom.), 5.08-4.93 (m, 18H, arCH $\mathrm{H}_{2} \mathrm{O}$ ), $4.90\left(\mathrm{~s}, 2 \mathrm{H}, \operatorname{arCH} \mathrm{H}_{2} \mathrm{O}\right), 4.88\left(\mathrm{~s}, 2 \mathrm{H}, \operatorname{arCH}_{2} \mathrm{O}\right), 4.84\left(\mathrm{~s}, 2 \mathrm{H}, \operatorname{arCH}_{2} \mathrm{O}\right), 4.36\left(\mathrm{~s}, 2 \mathrm{H}, \operatorname{arCH} \mathrm{Br}_{2}\right){ }^{13} \mathrm{C} \mathrm{NMR}(\delta$, ppm, $\mathrm{CDCl}_{3}$ ): 152.9, 152.7, 138.4, 137.9, 137.8, 137.7, 136.9, 136.8, 133.3, 133.2, 132.4, 128.4, 128.3, 128.0, 127.7, 127.4, 127.3, 109.1, 107.4, 106.7, 75.1, 71.4, 71.0, 70.8, 34.1. IR (n, cm $\left.{ }^{-1}, \mathrm{KBr}\right): 1588,1508$, 1245, 1127, 730. Anal. Calcd for $\mathrm{C}_{91} \mathrm{H}_{79} \mathrm{BrO}_{12}$ : C, 75.65; H, 5.52. Found: C, 74.40; H, 5.59. 
Supplement Table 1. Ratios of Averaged Principal Moments of the Radius of Gyration Tensor $\left(\left\langle L_{i}^{2}\right\rangle /\left\langle L_{1}^{2}\right\rangle\right)$, Relative Asphericities $\left(\langle b\rangle /\left\langle R_{g}^{2}\right\rangle\right)$ and Acylindricities $\left(\langle c\rangle /\left\langle R_{g}^{2}\right\rangle\right)$ Obtained for Reference Molecules .a

\begin{tabular}{|l|l|l|l|l|l|}
\hline Molecule & $\begin{array}{l}\text { Structural } \\
\text { type }\end{array}$ & $\frac{\left\langle L_{2}^{2}\right\rangle}{\left\langle L_{1}^{2}\right\rangle}$ & $\frac{\left\langle L_{3}^{2}\right\rangle}{\left\langle L_{1}^{2}\right\rangle}$ & $\frac{\langle b\rangle}{\left\langle R_{g}^{2}\right\rangle}$ & $\frac{\langle c\rangle}{\left\langle R_{g}^{2}\right\rangle}$ \\
\hline $\begin{array}{l}\text { n-Pentane } \\
\text { Lexaethynyl- } \\
\text { benzene }\end{array}$ & $\begin{array}{l}\text { Planar cycle } \\
\text { (Cylinder) }\end{array}$ & 0.52 & 0.49 & 0.25 & 0.02 \\
(Disk-star) & 0.94 & 0.14 & 0.22 & 0.39 \\
\hline & $\begin{array}{l}\text { Globular } \\
\text { Neopentane }\end{array}$ & 0.96 & 0.92 & 0.02 & 0.01 \\
\hline
\end{tabular}

${ }^{\mathrm{a}}$ For the definition of magnitudes $L_{i}^{2}$, asphericity $\mathbf{b}$, and acylindricity $\mathbf{c}$ see eqs. (6), (8), (10) and (11) of the main text. 
III. Representative spectra

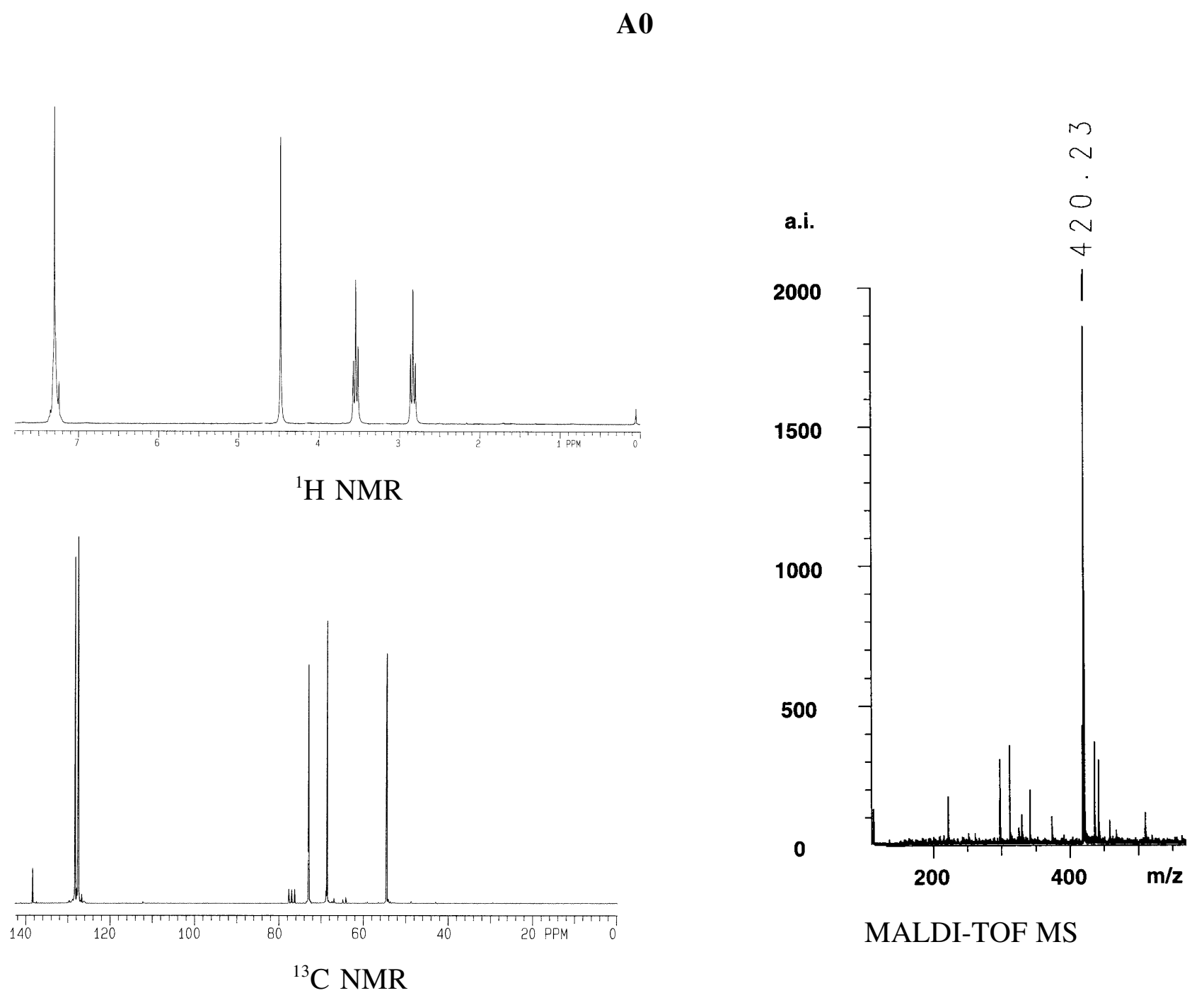


MA1
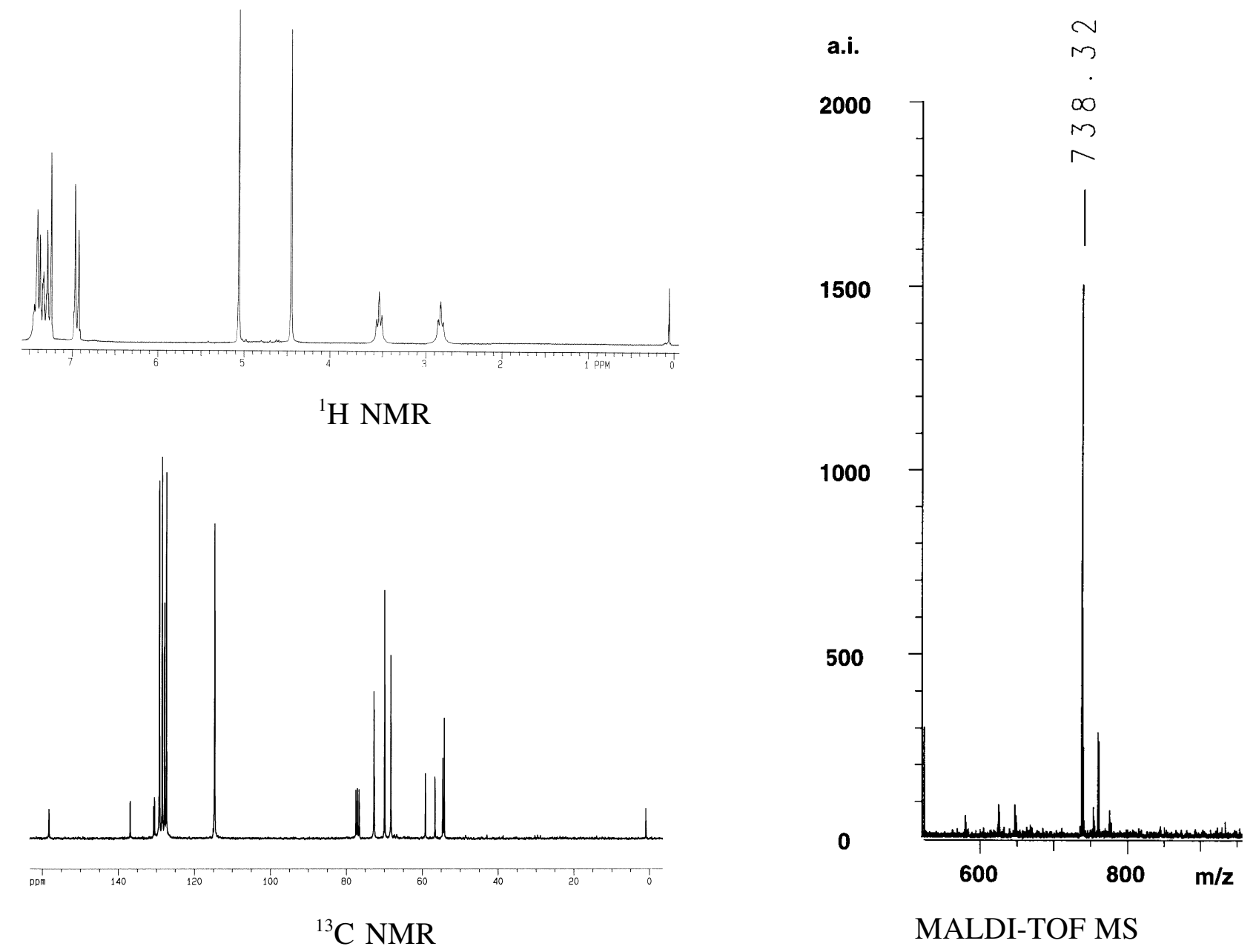
MA2
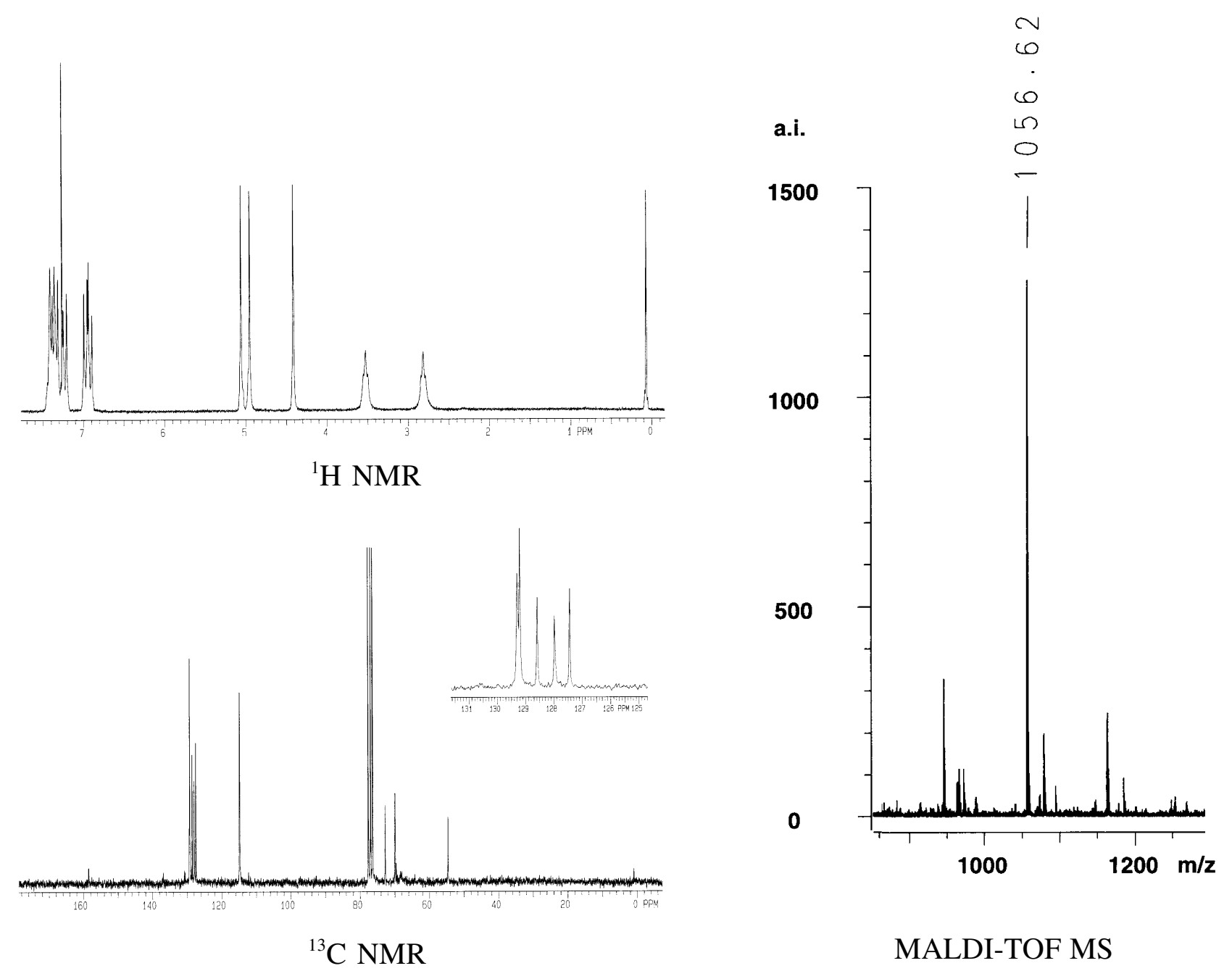

MALDI-TOF MS 
DA1

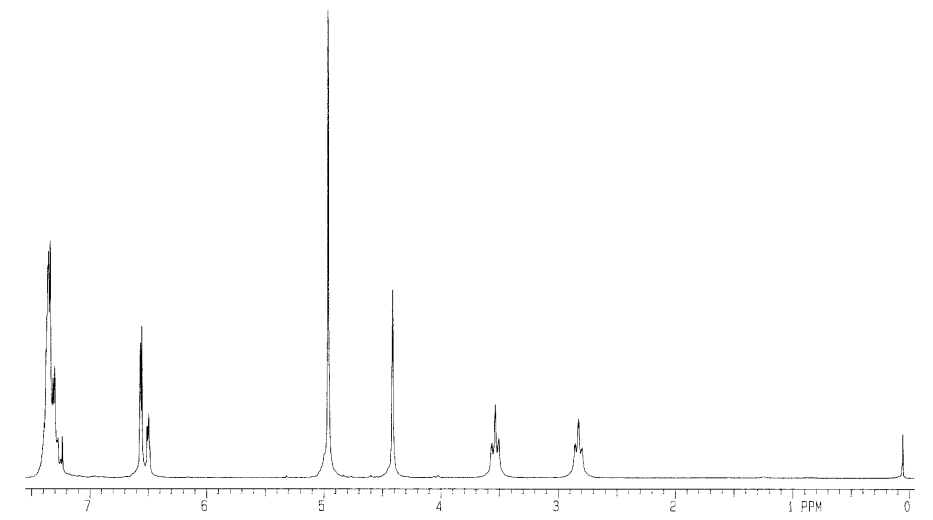

${ }^{1} \mathrm{H}$ NMR

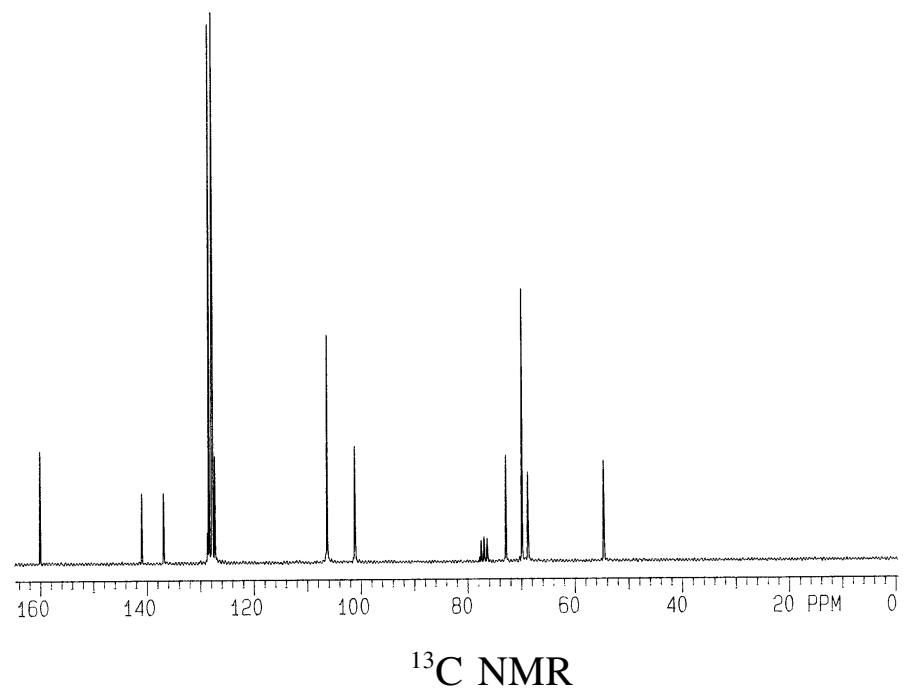

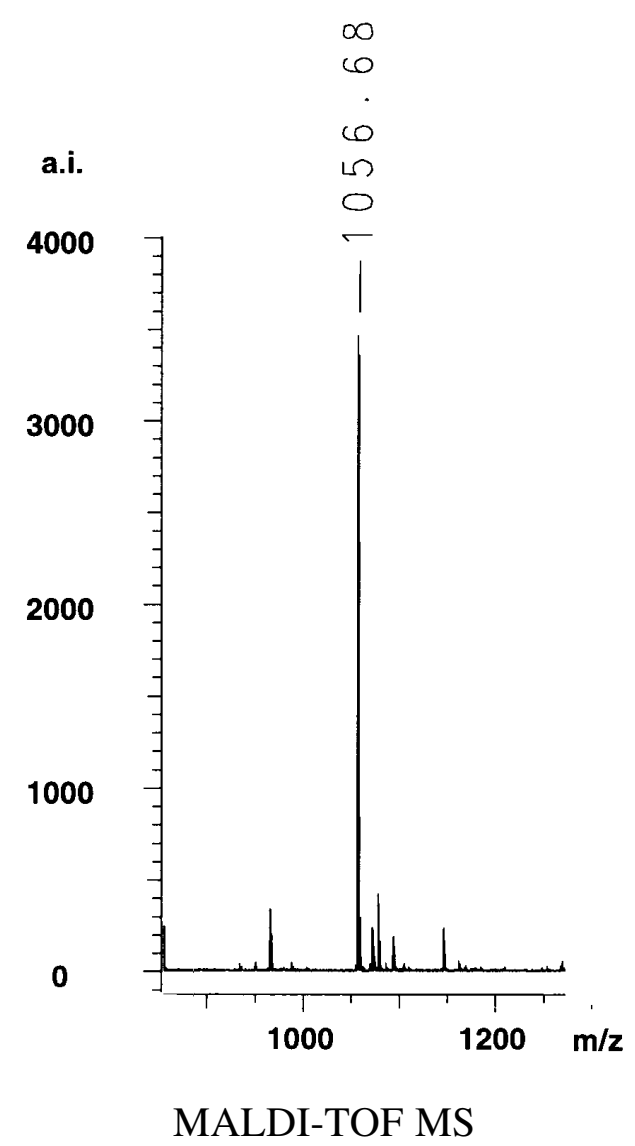


DA2
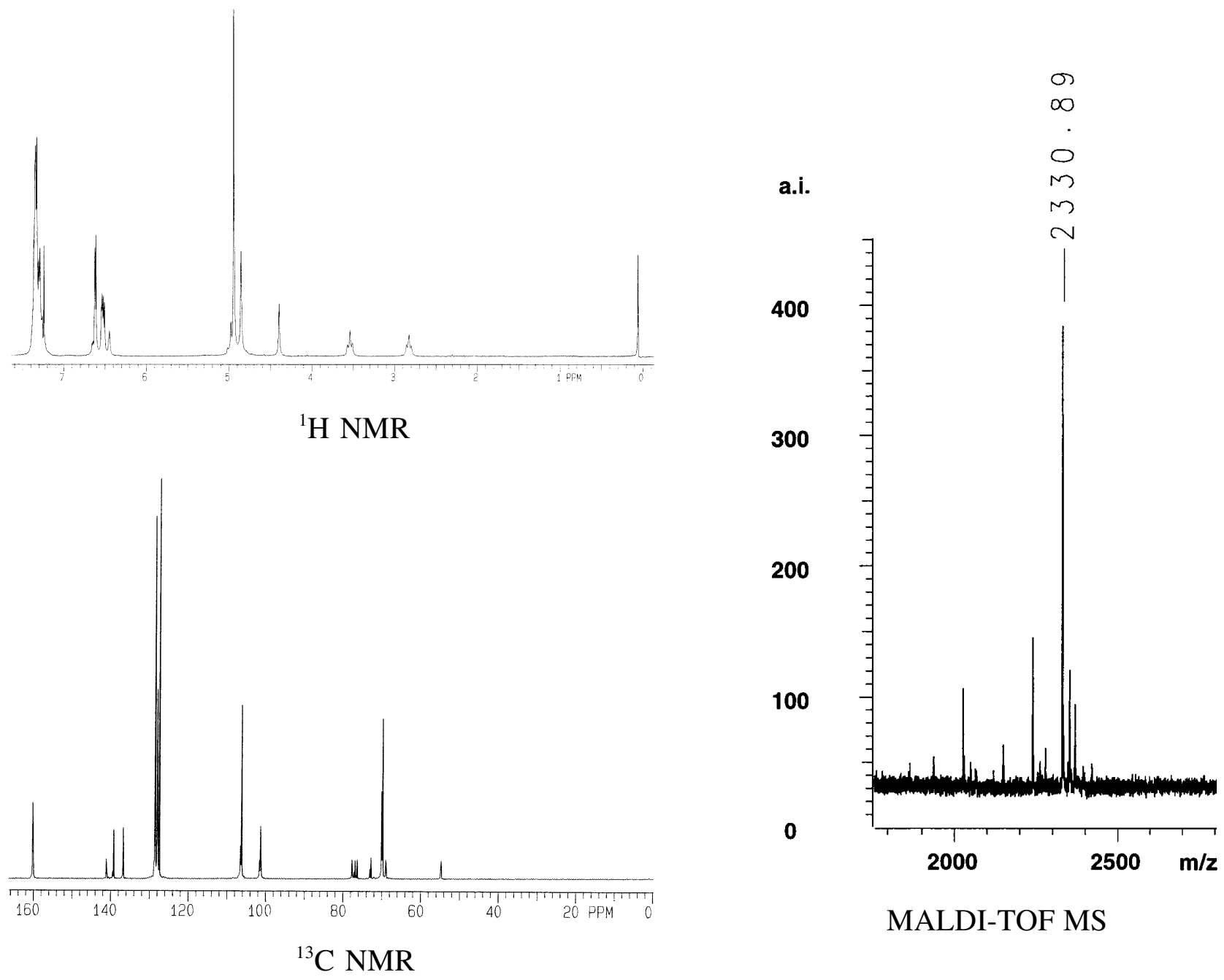

MALDI-TOF MS 
TA1

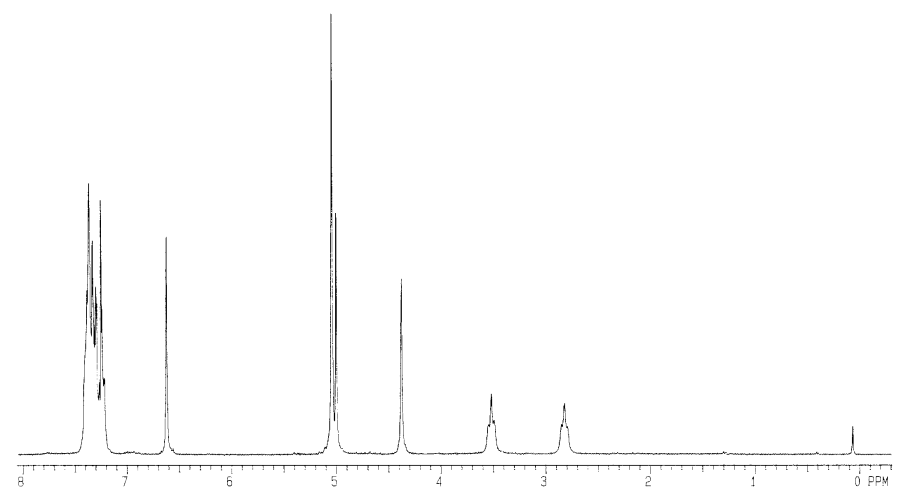

${ }^{1} \mathrm{H}$ NMR
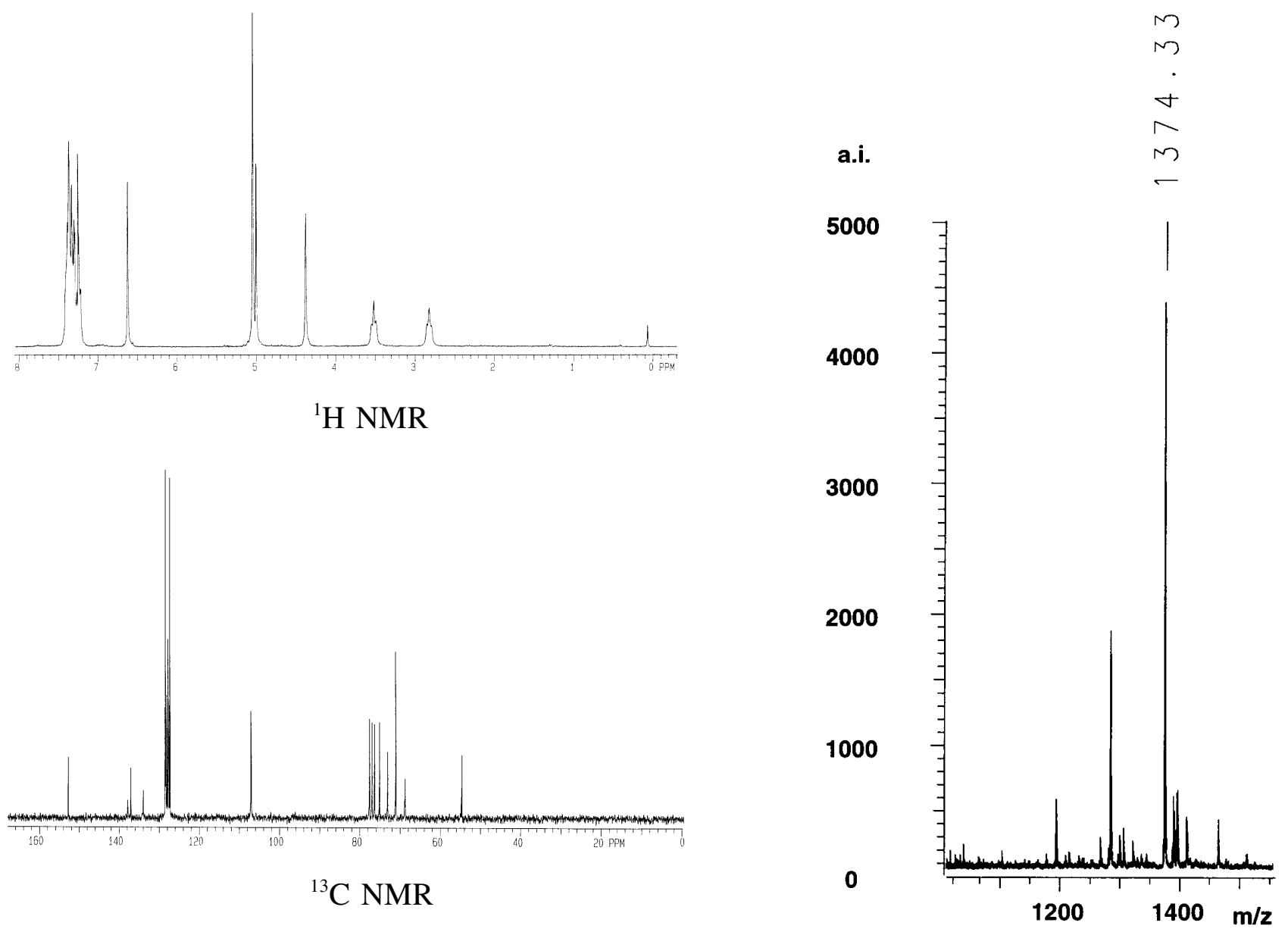

MALDI-TOF MS 
TA2
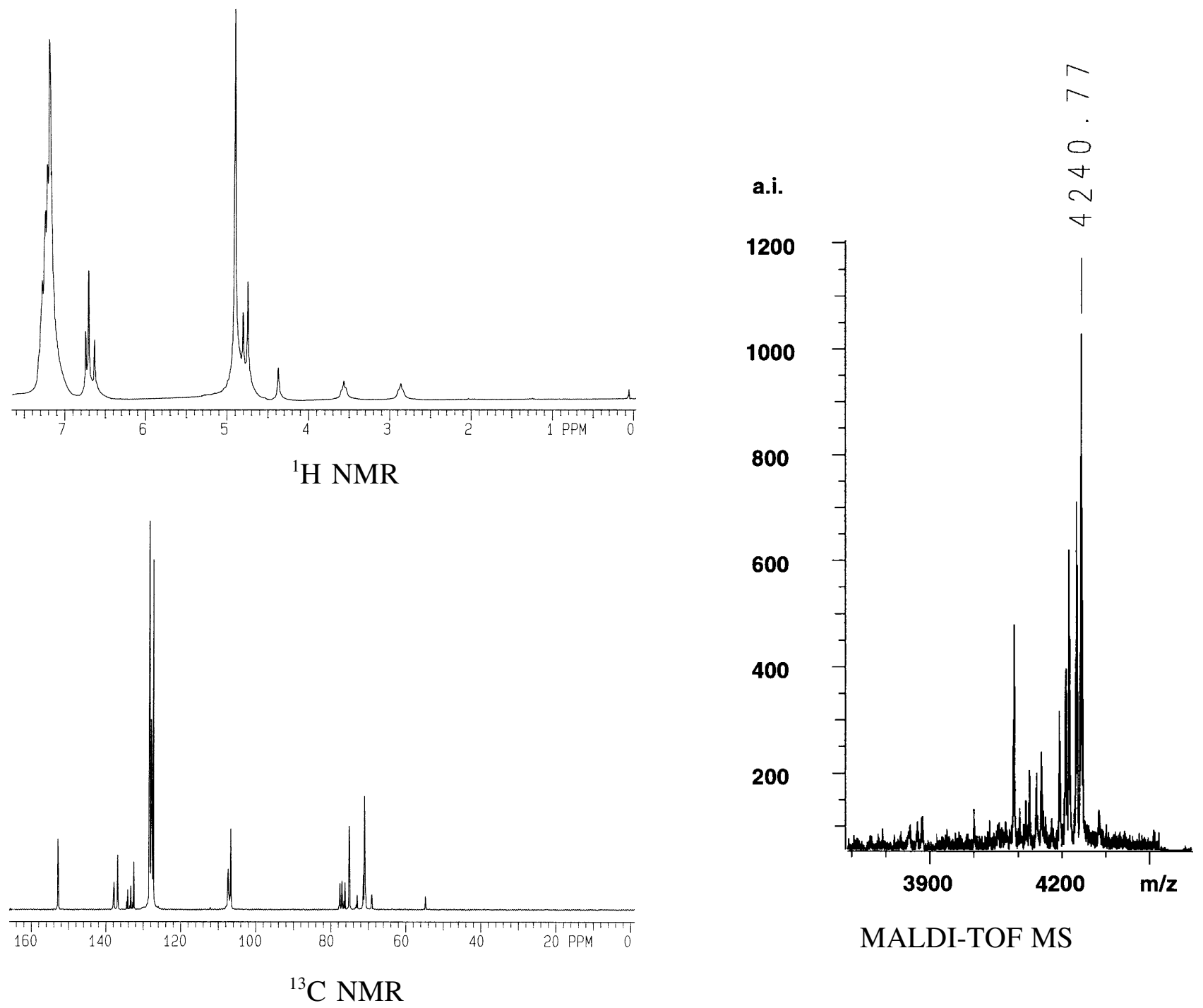
IV. Kinetic Measurements. Absorbance data measured for the reaction between benzaldehyde and nitroethanol catalyzed by amines A0, MA1-2, DA1-2 and TA1-2, according to the procedure described in the Experimental Section of the main text. All experiments were performed in triplicate. The pseudofirst order rate coefficients reported in Table 1 of the main text correspond to the average of these runs after the mathematical treatment described in eqs. (1)-(5) and in Figure 2 of the main text.

A0 (experiment 1) $\tau=7 \mathrm{~min}$

\begin{tabular}{|c|c|c|c|}
\hline $\mathrm{t}(\min )$ & $\mathrm{A}(\mathrm{C}=\mathrm{O})$ & $\mathrm{A}(\mathrm{C} \equiv \mathrm{N})$ & $\mathrm{A}(\mathrm{C}=\mathrm{O}) / \mathrm{A}(\mathrm{C} \equiv \mathrm{N})$ \\
\hline 0 & 5.0069 & 1.7987 & 2.7837 \\
\hline 7 & 4.3801 & 2.4983 & 1.7532 \\
\hline 14 & 3.9090 & 2.7372 & 1.4281 \\
\hline 21 & 3.5384 & 3.0255 & 1.1695 \\
\hline 28 & 2.9608 & 2.8687 & 1.0321 \\
\hline 35 & 2.4609 & 2.6749 & 0.9199 \\
\hline 42 & 2.1875 & 2.4298 & 0.9003 \\
\hline 49 & 2.3757 & 2.7055 & 0.8781 \\
\hline
\end{tabular}

A0 (experiment 2) $\tau=7 \mathrm{~min}$

\begin{tabular}{|c|c|c|c|}
\hline $\mathrm{t}(\min )$ & $\mathrm{A}(\mathrm{C}=\mathrm{O})$ & $\mathrm{A}(\mathrm{C} \equiv \mathrm{N})$ & $\mathrm{A}(\mathrm{C}=\mathrm{O}) / \mathrm{A}(\mathrm{C} \equiv \mathrm{N})$ \\
\hline 0 & 4.85232 & 1.83256 & 2.6478 \\
\hline 7 & 4.55918 & 2.35987 & 1.9320 \\
\hline 14 & 3.57346 & 2.39355 & 1.4929 \\
\hline 21 & 2.01022 & 1.54616 & 1.3001 \\
\hline 28 & 4.04764 & 3.29289 & 1.2292 \\
\hline 35 & 4.12039 & 3.64599 & 1.0196 \\
\hline 42 & 2.80925 & 2.75520 & 0.9601 \\
\hline 49 & 2.98387 & 3.10798 & 0.9201 \\
\hline 56 & 2.45472 & 2.66793 & \\
\hline
\end{tabular}

A0 (experiment 3 ) $\tau=7$ min

\begin{tabular}{|c|c|c|c|}
\hline $\mathrm{t}(\min )$ & $\mathrm{A}(\mathrm{C}=\mathrm{O})$ & $\mathrm{A}(\mathrm{C} \equiv \mathrm{N})$ & $\mathrm{A}(\mathrm{C}=\mathrm{O}) / \mathrm{A}(\mathrm{C} \equiv \mathrm{N})$ \\
\hline 0 & 4.34808 & 1.38539 & 3.1385 \\
\hline
\end{tabular}




\begin{tabular}{|c|c|c|c|}
\hline 7 & 4.58316 & 2.08977 & 2.1931 \\
\hline 14 & 2.97898 & 1.78826 & 1.6659 \\
\hline 21 & 4.5971 & 2.94793 & 1.5594 \\
\hline 28 & 4.20439 & 3.19308 & 1.3167 \\
\hline 35 & 3.36922 & 2.88331 & 1.1685 \\
\hline 42 & 2.30839 & 2.16487 & 1.0663 \\
\hline 56 & 3.41864 & 3.45623 & 0.99572 \\
\hline 63 & 3.77251 & 3.78874 & 0.98912 \\
\hline
\end{tabular}

MA1 (experiment 1) $\tau=6 \mathrm{~min}$

\begin{tabular}{|c|c|c|c|}
\hline $\mathrm{t}(\mathrm{min})$ & $\mathrm{A}(\mathrm{C}=\mathrm{O})$ & $\mathrm{A}(\mathrm{C} \equiv \mathrm{N})$ & $\mathrm{A}(\mathrm{C}=\mathrm{O}) / \mathrm{A}(\mathrm{C} \equiv \mathrm{N})$ \\
\hline 0 & 3.262 & 1.167 & 2.796 \\
\hline 1.5 & 7.147 & 2.654 & 2.693 \\
\hline 3 & 5.959 & 2.508 & 2.302 \\
\hline 4.5 & 4.928 & 2.416 & 2.040 \\
\hline 6 & 5.225 & 3.044 & 1.717 \\
\hline 7.5 & 3.148 & 2.079 & 1.514 \\
\hline 9 & 2.432 & 1.788 & 1.361 \\
\hline 10.5 & 1.631 & 1.345 & 1.212 \\
\hline 12 & 1.712 & 1.430 & 1.198 \\
\hline 13.5 & 0.986 & 0.940 & 1.049 \\
\hline 15 & 0.971 & 0.965 & 1.006 \\
\hline 16.5 & 1.775 & 1.848 & 0.961 \\
\hline 18 & 0.915 & 0.989 & 0.925 \\
\hline
\end{tabular}

MA1 (experiment 2) $\tau=6 \mathrm{~min}$

\begin{tabular}{|c|c|c|c|}
\hline $\mathrm{t}(\min )$ & $\mathrm{A}(\mathrm{C}=\mathrm{O})$ & $\mathrm{A}(\mathrm{C} \equiv \mathrm{N})$ & $\mathrm{A}(\mathrm{C}=\mathrm{O}) / \mathrm{A}(\mathrm{C} \equiv \mathrm{N})$ \\
\hline 0 & 7.9619 & 2.9154 & 2.731 \\
\hline 1.5 & 4.5310 & 1.7189 & 2.636 \\
\hline 3 & 5.3164 & 2.4387 & 2.180 \\
\hline
\end{tabular}




\begin{tabular}{|c|c|c|c|}
\hline 4.5 & 4.1319 & 2.1176 & 1.951 \\
\hline 6 & 2.6304 & 1.3565 & 1.939 \\
\hline 7.5 & 2.0753 & 1.2074 & 1.719 \\
\hline 9 & 3.8253 & 2.2635 & 1.690 \\
\hline 10.5 & 2.7842 & 2.1351 & 1.304 \\
\hline 12 & 1.5246 & 1.1040 & 1.381 \\
\hline 13.5 & 2.4768 & 1.8347 & 1.161 \\
\hline 15 & 1.1020 & 0.9492 & 0.927 \\
\hline 16.5 & 1.3832 & 1.4921 & 0.913 \\
\hline 18 & 0.5875 & 0.6435 & \\
\hline
\end{tabular}

MA1 (experiment 3) $\tau=6 \mathrm{~min}$

\begin{tabular}{|c|c|c|c|}
\hline $\mathrm{t}(\min )$ & $\mathrm{A}(\mathrm{C}=\mathrm{O})$ & $\mathrm{A}(\mathrm{C} \equiv \mathrm{N})$ & $\mathrm{A}(\mathrm{C}=\mathrm{O}) / \mathrm{A}(\mathrm{C} \equiv \mathrm{N})$ \\
\hline 0 & 6.7162 & 1.8934 & 3.5472 \\
\hline 1.5 & 6.0273 & 2.0541 & 2.9343 \\
\hline 3 & 6.7949 & 2.6283 & 2.5853 \\
\hline 4.5 & 3.6801 & 1.5537 & 2.3686 \\
\hline 6 & 6.1259 & 2.8611 & 2.0135 \\
\hline 7.5 & 2.2658 & 1.1253 & 1.8146 \\
\hline 9 & 1.4049 & 0.7742 & 1.6891 \\
\hline 10.5 & 4.0448 & 2.4205 & 1.5664 \\
\hline 12 & 2.5382 & 1.6204 & 1.4533 \\
\hline 13.5 & 2.2789 & 1.5681 & 1.4221 \\
\hline 15 & 3.3412 & 2.3495 & 1.3686 \\
\hline 16.5 & 1.1669 & 0.8526 & \\
\hline
\end{tabular}

MA2 (experiment 1) $\tau=15 \mathrm{~min}$

\begin{tabular}{|c|c|c|c|}
\hline $\mathrm{t}(\min )$ & $\mathrm{A}(\mathrm{C}=\mathrm{O})$ & $\mathrm{A}(\mathrm{C} \equiv \mathrm{N})$ & $\mathrm{A}(\mathrm{C}=\mathrm{O}) / \mathrm{A}(\mathrm{C} \equiv \mathrm{N})$ \\
\hline 0 & 4.014 & 1.990 & 2.017 \\
\hline 1.5 & 3.602 & 1.427 & 2.524 \\
\hline
\end{tabular}




\begin{tabular}{|c|c|c|c|}
\hline 3 & 6.515 & 2.345 & 2.778 \\
\hline 4.5 & 4.838 & 1.885 & 2.567 \\
\hline 6 & 7.955 & 3.106 & 2.561 \\
\hline 7.5 & 3.718 & 1.636 & 2.273 \\
\hline 9 & 5.245 & 2.189 & 2.396 \\
\hline 10.5 & 4.376 & 1.896 & 2.308 \\
\hline 12 & 1.973 & 0.905 & 2.179 \\
\hline 13.5 & 3.137 & 1.486 & 2.110 \\
\hline 15 & 2.952 & 1.424 & 2.073 \\
\hline 16.5 & 1.596 & 0.809 & 1.974 \\
\hline 18 & 2.238 & 1.195 & 1.873 \\
\hline 19.5 & 4.436 & 2.289 & 1.938 \\
\hline 21 & 2.530 & 1.420 & 1.781 \\
\hline 22.5 & 1.322 & 0.743 & 1.781 \\
\hline 31.5 & 1.350 & 0.941 & 1.435 \\
\hline 42 & 2.455 & 1.907 & 1.287 \\
\hline
\end{tabular}

MA2 (experiment 2) $\tau=15 \min$

\begin{tabular}{|c|c|c|c|}
\hline $\mathrm{t}(\mathrm{min})$ & $\mathrm{A}(\mathrm{C}=\mathrm{O})$ & $\mathrm{A}(\mathrm{C} \equiv \mathrm{N})$ & $\mathrm{A}(\mathrm{C}=\mathrm{O}) / \mathrm{A}(\mathrm{C} \equiv \mathrm{N})$ \\
\hline 0 & 8.013 & 2.905 & 2.759 \\
\hline 1.5 & 5.013 & 1.981 & 2.531 \\
\hline 3 & 2.596 & 1.037 & 2.503 \\
\hline 4.5 & 3.041 & 1.230 & 2.472 \\
\hline 6 & 5.658 & 2.406 & 2.351 \\
\hline 7.5 & 1.794 & 0.835 & 2.148 \\
\hline 9 & 1.910 & 0.885 & 2.159 \\
\hline 10.5 & 1.184 & 1.004 & 1.179 \\
\hline 12 & 3.025 & 1.630 & 1.855 \\
\hline 13.5 & 2.119 & 1.132 & 1.871 \\
\hline 15 & 3.173 & 1.711 & 1.855 \\
\hline
\end{tabular}




\begin{tabular}{|c|c|c|c|}
\hline 16.5 & 6.323 & 3.593 & 1.760 \\
\hline 18 & 2.482 & 1.289 & 1.925 \\
\hline 19.5 & 5.804 & 3.158 & 1.838 \\
\hline 21 & 2.782 & 1.764 & 1.577 \\
\hline 22.5 & 2.201 & 1.443 & 1.525 \\
\hline 24 & 3.739 & 2.476 & 1.510 \\
\hline 25.5 & 3.510 & 2.359 & 1.488 \\
\hline 27 & 4.464 & 3.064 & 1.457 \\
\hline 28.5 & 3.486 & 2.490 & 1.400 \\
\hline 39 & 1.706 & 1.475 & 1.156 \\
\hline
\end{tabular}

MA2 (experiment 3) $\tau=15 \mathrm{~min}$

\begin{tabular}{|c|c|c|c|}
\hline $\mathrm{t}(\min )$ & $\mathrm{A}(\mathrm{C}=\mathrm{O})$ & $A(C \equiv N)$ & $\mathrm{A}(\mathrm{C}=\mathrm{O}) / \mathrm{A}(\mathrm{C} \equiv \mathrm{N})$ \\
\hline 0 & 2.578 & 1.050 & 2.456 \\
\hline 1.5 & 7.854 & 3.209 & 2.447 \\
\hline 3 & 7.588 & 2.924 & 2.595 \\
\hline 4.5 & 2.402 & 0.974 & 2.466 \\
\hline 6 & 3.371 & 1.505 & 2.240 \\
\hline 7.5 & 6.193 & 2.600 & 2.382 \\
\hline 9 & 2.803 & 1.364 & 2.055 \\
\hline 10.5 & 12.421 & 5.391 & 2.304 \\
\hline 12 & 3.628 & 1.869 & 1.942 \\
\hline 13.5 & 6.466 & 3.282 & 1.970 \\
\hline 15 & 4.681 & 2.586 & 1.810 \\
\hline 16.5 & 3.221 & 1.788 & 1.801 \\
\hline 18 & 5.909 & 3.380 & 1.748 \\
\hline 19.5 & 2.748 & 1.620 & 1.696 \\
\hline 21 & 2.237 & 1.418 & 1.577 \\
\hline 22.5 & 2.676 & 1.715 & 1.560 \\
\hline 24 & 4.711 & 3.044 & 1.548 \\
\hline
\end{tabular}




\begin{tabular}{|c|c|c|c|}
\hline 25.5 & 3.279 & 2.213 & 1.481 \\
\hline 27 & 2.683 & 1.861 & 1.442 \\
\hline 28.5 & 3.342 & 2.284 & 1.463 \\
\hline 30 & 1.671 & 1.199 & 1.394 \\
\hline 33.5 & 4.930 & 3.550 & 1.389 \\
\hline 36.5 & 8.449 & 6.090 & 1.387 \\
\hline 41 & 11.708 & 8.492 & 1.379 \\
\hline 45 & 9.265 & 7.390 & 1.254 \\
\hline 49 & 1.594 & 1.160 & 1.374 \\
\hline 53 & 5.134 & 4.404 & 1.166 \\
\hline 57 & 6.988 & 6.348 & 1.101 \\
\hline
\end{tabular}

DA1 (experiment 1) $\tau=10 \mathrm{~min}$

\begin{tabular}{|c|c|c|c|}
\hline $\mathrm{t}(\mathrm{min})$ & $\mathrm{A}(\mathrm{C}=\mathrm{O})$ & $\mathrm{A}(\mathrm{C} \equiv \mathrm{N})$ & $\mathrm{A}(\mathrm{C}=\mathrm{O}) / \mathrm{A}(\mathrm{C} \equiv \mathrm{N})$ \\
\hline 0 & 3.04359 & 1.19299 & 2.5512 \\
\hline 10 & 2.81836 & 1.54185 & 1.8279 \\
\hline 20 & 2.66255 & 2.00045 & 1.3310 \\
\hline 30 & 2.35995 & 1.92421 & 1.2264 \\
\hline 40 & 3.13332 & 3.01816 & 1.0382 \\
\hline 50 & 2.09895 & 2.22119 & 0.94497 \\
\hline 60 & 2.47934 & 2.67011 & 0.92859 \\
\hline 70 & 2.16475 & 2.39963 & 0.90212 \\
\hline 80 & 3.27266 & 3.50697 & 0.93319 \\
\hline 90 & 2.16259 & 2.43702 & 0.88739 \\
\hline 100 & 2.94507 & 3.37130 & 0.87357 \\
\hline 110 & 2.98831 & 3.38515 & 0.88277 \\
\hline
\end{tabular}

DA1 (experiment 2) $\tau=10 \mathrm{~min}$

\begin{tabular}{|c|c|c|c|}
\hline $\mathrm{t}(\min )$ & $\mathrm{A}(\mathrm{C}=\mathrm{O})$ & $\mathrm{A}(\mathrm{C} \equiv \mathrm{N})$ & $\mathrm{A}(\mathrm{C}=\mathrm{O}) / \mathrm{A}(\mathrm{C} \equiv \mathrm{N})$ \\
\hline 0 & 5.75989 & 2.13465 & 2.6983 \\
\hline
\end{tabular}




\begin{tabular}{|c|c|c|c|}
\hline 10 & 3.48050 & 1.97094 & 1.7659 \\
\hline 20 & 3.71679 & 2.46806 & 1.5060 \\
\hline 30 & 2.64951 & 2.18112 & 1.2148 \\
\hline 40 & 1.57794 & 1.50492 & 1.0485 \\
\hline 50 & 2.29406 & 2.35293 & 0.97498 \\
\hline 60 & 2.25840 & 2.41087 & 0.93676 \\
\hline 70 & 2.45333 & 2.72724 & 0.89957 \\
\hline 80 & 2.18127 & 2.46050 & 0.88651 \\
\hline 90 & 2.51945 & 2.82791 & 0.89792 \\
\hline 100 & 2.39136 & 2.69322 & 0.86498 \\
\hline 110 & 1.86622 & 2.15752 & 0.87509 \\
\hline 120 & 2.63768 & 3.01415 & 0.87903 \\
\hline 130 & 2.67412 & 3.04214 & \\
\hline
\end{tabular}

DA1 (experiment 3) $\tau=10 \mathrm{~min}$

\begin{tabular}{|c|c|c|c|}
\hline $\mathrm{t}(\mathrm{min})$ & $\mathrm{A}(\mathrm{C}=\mathrm{O})$ & $\mathrm{A}(\mathrm{C} \equiv \mathrm{N})$ & $\mathrm{A}(\mathrm{C}=\mathrm{O}) / \mathrm{A}(\mathrm{C} \equiv \mathrm{N})$ \\
\hline 0 & 3.6905 & 1.40201 & 2.6323 \\
\hline 10 & 4.17461 & 2.10794 & 1.9804 \\
\hline 20 & 4.52706 & 2.97215 & 1.5232 \\
\hline 30 & 3.84226 & 2.99883 & 1.0806 \\
\hline 40 & 2.38009 & 2.20265 & 1.0159 \\
\hline 50 & 3.25071 & 3.19973 & 0.94594 \\
\hline 60 & 2.28697 & 2.41768 & 0.92611 \\
\hline 70 & 2.83402 & 3.06014 & 0.88423 \\
\hline 80 & 3.42383 & 3.87212 & 0.84209 \\
\hline 90 & 2.26192 & 2.68605 & 0.85262 \\
\hline 100 & 2.63916 & 3.09537 & \\
\hline
\end{tabular}

DA2 (experiment 1) $\tau=120 \mathrm{~min}$

\begin{tabular}{|l|l|l|l|}
$\mathrm{t}(\mathrm{min})$ & $\mathrm{A}(\mathrm{C}=\mathrm{O})$ & $\mathrm{A}(\mathrm{C} \equiv \mathrm{N})$ & $\mathrm{A}(\mathrm{C}=\mathrm{O}) / \mathrm{A}(\mathrm{C} \equiv \mathrm{N})$ \\
\hline
\end{tabular}




\begin{tabular}{|c|c|c|c|}
\hline 0 & 8.25054 & 2.48053 & 3.3261 \\
\hline 15 & 5.52098 & 2.17266 & 2.5411 \\
\hline 30 & 4.17683 & 1.94604 & 2.1463 \\
\hline 45 & 3.92791 & 2.09925 & 1.8711 \\
\hline 60 & 3.68042 & 2.26260 & 1.6266 \\
\hline 75 & 3.09701 & 2.10243 & 1.4731 \\
\hline 90 & 3.32376 & 2.45446 & 1.3542 \\
\hline 105 & 3.74275 & 2.91624 & 1.2390 \\
\hline 120 & 3.28882 & 2.65433 & 1.1615 \\
\hline 135 & 2.76746 & 2.38267 & 1.1747 \\
\hline 150 & 2.94258 & 2.50487 & 1.1101 \\
\hline 165 & 2.07124 & 1.86576 & 1.1123 \\
\hline 180 & 2.79688 & 2.51447 & \\
\hline
\end{tabular}

DA2 (experiment 2) $\tau=120 \mathrm{~min}$

\begin{tabular}{|c|c|c|c|}
\hline $\mathrm{t}(\mathrm{min})$ & $\mathrm{A}(\mathrm{C}=\mathrm{O})$ & $\mathrm{A}(\mathrm{C} \equiv \mathrm{N})$ & $\mathrm{A}(\mathrm{C}=\mathrm{O}) / \mathrm{A}(\mathrm{C} \equiv \mathrm{N})$ \\
\hline 0 & 7.50928 & 2.19117 & 3.4271 \\
\hline 15 & 5.08612 & 1.9860 & 2.5610 \\
\hline 30 & 3.61277 & 1.6985 & 2.1270 \\
\hline 45 & 3.32516 & 1.82208 & 1.8249 \\
\hline 60 & 2.23781 & 1.42138 & 1.4131 \\
\hline 75 & 2.67456 & 1.89264 & 1.3294 \\
\hline 90 & 2.68677 & 2.02102 & 1.2291 \\
\hline 105 & 2.05447 & 1.67149 & 1.1683 \\
\hline 120 & 1.58842 & 1.35955 & \\
\hline 135 & 1.77594 & 1.54803 & \\
\hline
\end{tabular}

DA2 (experiment 3) $\tau=120 \mathrm{~min}$

\begin{tabular}{|c|c|c|c|}
\hline $\mathrm{t}(\min )$ & $\mathrm{A}(\mathrm{C}=\mathrm{O})$ & $\mathrm{A}(\mathrm{C} \equiv \mathrm{N})$ & $\mathrm{A}(\mathrm{C}=\mathrm{O}) / \mathrm{A}(\mathrm{C} \equiv \mathrm{N})$ \\
\hline 0 & 6.43065 & 2.08087 & 3.0904 \\
\hline
\end{tabular}




\begin{tabular}{|l|l|l|l|}
\hline 15 & 5.65080 & 2.26201 & 2.4981 \\
\hline 30 & 5.2591 & 2.38953 & 2.2009 \\
\hline 45 & 3.57149 & 1.79045 & 1.9947 \\
\hline 60 & 2.45131 & 1.51370 & 1.6194 \\
\hline 75 & 3.32380 & 2.47173 & 1.3447 \\
\hline 90 & 2.72764 & 2.00130 & 1.3629 \\
\hline 105 & 2.71081 & 2.1718 & 1.2482 \\
\hline 120 & 1.62154 & 1.39732 & 1.1605 \\
\hline 135 & 2.33016 & 2.02141 & 1.1527 \\
\hline 150 & 1.7210 & 1.52623 & 1.1276 \\
\hline 165 & 1.31938 & 1.20152 & 1.0981 \\
\hline 180 & 2.12060 & 1.96778 & 1.0777 \\
\hline 195 & 1.33939 & 1.24616 & 1.0748 \\
\hline 210 & 2.48274 & 2.29746 & 1.0806 \\
\hline 225 & 2.21992 & 2.12268 & 1.0458 \\
\hline
\end{tabular}

TA1 (experiment 1) $\tau=110 \mathrm{~min}$

\begin{tabular}{|c|c|c|c|}
\hline $\mathrm{t}(\mathrm{min})$ & $\mathrm{A}(\mathrm{C}=\mathrm{O})$ & $\mathrm{A}(\mathrm{C} \equiv \mathrm{N})$ & $\mathrm{A}(\mathrm{C}=\mathrm{O}) / \mathrm{A}(\mathrm{C} \equiv \mathrm{N})$ \\
\hline 0 & 9.62942 & 2.89697 & 3.3240 \\
\hline 10 & 8.97884 & 3.1571 & 2.8440 \\
\hline 20 & 6.23523 & 2.49702 & 2.4971 \\
\hline 30 & 7.54142 & 3.07663 & 2.4512 \\
\hline 40 & 8.64728 & 3.80366 & 2.2734 \\
\hline 50 & 6.48350 & 2.92477 & 2.2167 \\
\hline 60 & 8.93930 & 4.08476 & 2.1885 \\
\hline 70 & 8.93930 & 4.08476 & 2.0400 \\
\hline 80 & 8.5453 & 4.29724 & 1.9885 \\
\hline 90 & 6.23604 & 3.49871 & 1.7824 \\
\hline 100 & 7.72484 & 4.46188 & 1.7313 \\
\hline 110 & 4.60459 & 2.94254 & 1.5648 \\
\hline
\end{tabular}




\begin{tabular}{|l|l|l|l|}
\hline 120 & 7.89434 & 4.64120 & 1.7009 \\
\hline 130 & 7.21725 & 4.71421 & 1.5310 \\
\hline 140 & 6.44873 & 4.30457 & 1.4981 \\
\hline 150 & 5.10281 & 3.73402 & 1.3666 \\
\hline 160 & 7.16024 & 3.60596 & 1.9857 \\
\hline 170 & 4.28485 & 3.41433 & 1.2550 \\
\hline 180 & 2.68886 & 2.31991 & 1.1590 \\
\hline 190 & 3.33507 & 2.81046 & 1.1867 \\
\hline 200 & 2.44868 & 2.16370 & 1.1317 \\
\hline 210 & 4.41760 & 3.89806 & 1.1333 \\
\hline 220 & 4.32763 & 3.99551 & 1.0831 \\
\hline 230 & 4.21216 & 3.83737 & 1.0977 \\
\hline
\end{tabular}

TA1 (experiment 2) $\tau=110 \mathrm{~min}$

\begin{tabular}{|c|c|c|c|}
\hline $\mathrm{t}(\mathrm{min})$ & $\mathrm{A}(\mathrm{C}=\mathrm{O})$ & $\mathrm{A}(\mathrm{C} \equiv \mathrm{N})$ & $\mathrm{A}(\mathrm{C}=\mathrm{O}) / \mathrm{A}(\mathrm{C} \equiv \mathrm{N})$ \\
\hline 0 & 2.04272 & 0.697329 & 2.9464 \\
\hline 10 & 10.6651 & 3.67985 & 2.8982 \\
\hline 20 & 9.12355 & 3.68572 & 2.4754 \\
\hline 30 & 8.41626 & 3.67064 & 2.2929 \\
\hline 40 & 6.16838 & 3.02568 & 2.0387 \\
\hline 50 & 7.23902 & 3.80759 & 1.9012 \\
\hline 60 & 5.49373 & 3.23578 & 1.6978 \\
\hline 70 & 8.60611 & 4.97323 & 1.7305 \\
\hline 80 & 7.58179 & 4.77566 & 1.5876 \\
\hline 90 & 7.06461 & 4.79829 & 1.4723 \\
\hline 100 & 4.80587 & 3.61726 & 1.3286 \\
\hline 110 & 6.44678 & 4.84545 & 1.3305 \\
\hline 120 & 4.82525 & 3.73929 & 1.2904 \\
\hline 130 & 3.92867 & 3.28408 & 1.1963 \\
\hline 140 & 5.30982 & 4.63635 & 1.1453 \\
\hline
\end{tabular}




\begin{tabular}{|c|c|c|c|}
\hline 150 & 4.96106 & 4.37210 & 1.1347 \\
\hline 160 & 6.12105 & 5.75805 & 1.0630 \\
\hline 170 & 2.16299 & 2.00697 & 1.0777 \\
\hline 180 & 0.88077 & 0.84595 & 1.0412 \\
\hline 190 & 1.31251 & 1.27204 & 1.0318 \\
\hline 200 & 0.86708 & 0.84945 & 1.0208 \\
\hline 210 & 1.05722 & 1.04942 & 1.0074 \\
\hline 220 & 1.6020 & 1.56290 & 1.0250 \\
\hline
\end{tabular}

TA1 (experiment 3) $\tau=110 \mathrm{~min}$

\begin{tabular}{|c|c|c|c|}
\hline $\mathrm{t}(\mathrm{min})$ & $\mathrm{A}(\mathrm{C}=\mathrm{O})$ & $\mathrm{A}(\mathrm{C} \equiv \mathrm{N})$ & $\mathrm{A}(\mathrm{C}=\mathrm{O}) / \mathrm{A}(\mathrm{C} \equiv \mathrm{N})$ \\
\hline 0 & 8.96941 & 3.15202 & 2.8456 \\
\hline 10 & 5.00489 & 1.83275 & 2.7308 \\
\hline 20 & 5.63514 & 2.24761 & 2.5072 \\
\hline 30 & 10.1458 & 3.94669 & 2.5707 \\
\hline 40 & 5.70878 & 2.86346 & 1.9937 \\
\hline 60 & 2.83768 & 1.64704 & 1.9229 \\
\hline 70 & 9.45209 & 5.17344 & 1.8270 \\
\hline 80 & 5.91519 & 3.45990 & 1.7096 \\
\hline 90 & 4.38695 & 2.75239 & 1.5939 \\
\hline 100 & 4.76441 & 3.05367 & 1.5602 \\
\hline 110 & 5.54045 & 3.81588 & 1.4519 \\
\hline 120 & 5.18494 & 3.77824 & 1.3723 \\
\hline 130 & 4.38557 & 3.35035 & 1.3026 \\
\hline 140 & 5.61319 & 4.30963 & 1.3025 \\
\hline 150 & 3.78202 & 3.16312 & 1.1957 \\
\hline 160 & 4.38773 & 3.73178 & 1.1758 \\
\hline 170 & 4.39672 & 3.81218 & 1.1533 \\
\hline 180 & 5.32519 & 4.58702 & 1.16093 \\
\hline & & & \\
\hline & & & \\
\hline
\end{tabular}

TA2 (experiment 1) $\tau=120 \mathrm{~min}$ 


\begin{tabular}{|c|c|c|c|}
\hline $\mathrm{t}(\mathrm{min})$ & $\mathrm{A}(\mathrm{C}=\mathrm{O})$ & $\mathrm{A}(\mathrm{C} \equiv \mathrm{N})$ & $\mathrm{A}(\mathrm{C}=\mathrm{O}) / \mathrm{A}(\mathrm{C} \equiv \mathrm{N})$ \\
\hline 0 & 2.914 & 0.979 & 2.977 \\
\hline 60 & 1.889 & 0.848 & 2.227 \\
\hline 120 & 1.137 & 0.585 & 1.942 \\
\hline 180 & 1.518 & 1.060 & 1.432 \\
\hline 240 & 1.490 & 1.144 & 1.302 \\
\hline 300 & 1.203 & 1.010 & 1.191 \\
\hline 360 & 2.169 & 1.923 & 1.128 \\
\hline 420 & 4.098 & 3.987 & 1.028 \\
\hline
\end{tabular}

TA2 (experiment 2) $\tau=120 \mathrm{~min}$

\begin{tabular}{|c|c|c|c|}
\hline $\mathrm{t}(\mathrm{min})$ & $\mathrm{A}(\mathrm{C}=\mathrm{O})$ & $\mathrm{A}(\mathrm{C} \equiv \mathrm{N})$ & $\mathrm{A}(\mathrm{C}=\mathrm{O}) / \mathrm{A}(\mathrm{C} \equiv \mathrm{N})$ \\
\hline 0 & 9.56394 & 3.42680 & 2.7909 \\
\hline 30 & 3.67507 & 1.74371 & 2.1076 \\
\hline 60 & 3.41736 & 2.01046 & 1.6998 \\
\hline 90 & 3.80746 & 2.46092 & 1.5472 \\
\hline 120 & 2.56047 & 1.79769 & 1.4243 \\
\hline 150 & 4.74523 & 3.67509 & 1.2912 \\
\hline 180 & 4.50045 & 3.91618 & 1.1492 \\
\hline 210 & 2.64612 & 2.47453 & 1.0693 \\
\hline 240 & 2.67431 & 2.69312 & 0.99301 \\
\hline 270 & 2.24028 & 2.23461 & 1.0025 \\
\hline
\end{tabular}

TA2 (experiment 3) $\tau=135 \mathrm{~min}$

\begin{tabular}{|c|c|c|c|}
\hline $\mathrm{t}(\mathrm{min})$ & $\mathrm{A}(\mathrm{C}=\mathrm{O})$ & $\mathrm{A}(\mathrm{C} \equiv \mathrm{N})$ & $\mathrm{A}(\mathrm{C}=\mathrm{O}) / \mathrm{A}(\mathrm{C} \equiv \mathrm{N})$ \\
\hline 0 & 8.09405 & 2.71504 & 2.9812 \\
\hline 45 & 2.71317 & 1.33436 & 2.0333 \\
\hline 90 & 3.09998 & 1.71066 & 1.8122 \\
\hline 135 & 3.21451 & 1.79329 & 1.7925 \\
\hline 180 & 2.64289 & 1.85496 & 1.4248 \\
\hline
\end{tabular}




\begin{tabular}{|c|c|c|c|}
\hline 225 & 3.12916 & 2.40381 & 1.3018 \\
\hline 270 & 3.03009 & 2.28643 & 1.3252 \\
\hline 315 & 2.99342 & 2.56854 & 1.1654 \\
\hline 360 & 3.57513 & 3.11897 & 1.1463 \\
\hline 405 & 3.11738 & 3.05289 & 1.0211 \\
\hline 450 & 1.38757 & 1.58409 & 0.87594 \\
\hline 495 & 2.99088 & 2.73162 & 1.0949 \\
\hline 540 & 3.10752 & 3.07143 & 1.0118 \\
\hline 585 & 2.48496 & 2.55442 & 0.97281 \\
\hline
\end{tabular}

\section{References}

1. Armarego, W. L. F.; Perrin, D. D. Purification of Laboratory Chemicals; Butterworth-Heinemann: Oxford, 1996.

2. Kashdan, D. S.; Scharwtz, J. A.; Rapoport, H. J. Org. Chem. 1982, 47, 2638.

3. Hawker, C. J.; Fréchet, J. M. J.; J. Am. Chem. Soc. 1990, 112, 7638.

4. Eich, E.; Pertz, H.; Kaloga, M.; Schultz, J.; Fesen, M. R.; Mazumder, A.; Pommier, Y. J. Med. Chem. 1996, 39, 86.

5. Krishnamoortthy, K.; Ambade, A: V.; Mishra, S. P.; Kanungo, M.; Contractor, A: Q.; Kumar, A. Polymer 2002, 43, 6465. 